\title{
Lymphotoxin and the amazing technicolor circus of intestinal homeostasis
}

\author{
JL Browning ${ }^{1}$
}

\section{Recently a new lymphocyte subset called innate lymphoid cells has emerged and it includes key producers of interleukin (IL)-17 and IL-22 in the mucosal environment. Using Citrobacter rodentium infection to deliver a pathogenic insult to the colon, two studies have revealed an underlying role for lymphotoxin- $\beta$ receptor signaling in the generation of IL-22 by these cells. This observation links a system well known for its ability to organize lymphoid microenvironments into a basic mucosal response.}

The circus thrills the senses with unimaginable spectacles. By analogy, mucosal immunologists could identify the menagerie of participants going in and out of the circus tent, but have longed to view the show in its whole splendor. Over the last decade, the tent has been opening its doors revealing the amazing equilibrium between the immune system and commensal flora at the mucosal interface. The intestinal microenvironments need to establish homeostasis with the gut contents, yet respond, usually aggressively, to an imbalance or pathogen while preserving the ability of the gut to harvest nutrients. Having a key role in this Herculean task is a new class of lymphocytes called innate lymphoid cells (ILCs)..$^{1,2}$ One branch of this family is dependent upon the transcription factor ROR $\gamma \mathrm{t}$ for the development and includes the lymphoid tissue inducer cell (LTi), an "adult" LTi-like cell and a natural killer (NK) cell (NK22 or NKp46+ NK cell). Robust interleukin (IL)-17 or
IL-22 secretion can be a trademark of these cells, yet they lack surface T- or $\mathrm{B}$-cell receptors and hence are considered "innate". Both cytokines trigger complex spectra of activity with both protective and proinflammatory components that appear to vary from organ to organ. ${ }^{2} \mathrm{~A}$ second branch of ROR $\gamma \mathrm{t}$-independent ILC has been described on the basis of Th2-type cytokine secretion. Along with these discoveries, substantial progress has occurred in the understanding of the development and function of organized lymphoid environments. Adaptive immunological processes do not occur efficiently without such structures and in the gut they include the developmentally determined draining lymph nodes and Peyer's patches as well as the non-predetermined tertiary lymphoid tissues (TLTs), i.e., isolated lymphoid follicles (ILF), cryptopatches, and cecal and colonic lymphoid patches (CLP, historically referred to simply as colonic patches). ${ }^{3}$ It was into the intersection of these exciting systems that the membrane form of lymphotoxin (LT) was recently inserted (Figure 1).

In general, the LT system is a mechanism by which lymphocytes can instruct stromal cells to differentiate into specialized reticular networks and vasculature. These elements form the portals that gate lymphocyte entry into organized sites and the frameworks that position cells for optimal encounters with antigen. ${ }^{4}$ The LT motif is used in the gut during mesenteric lymph node and Peyer's patch development as well as to drive the generation and maturation of mucosal TLTs. Over a decade of work has linked the LT system to aspects of mucosal immunology, including $\mathrm{M}$-cell differentiation, $\gamma \delta$ T-cell differentiation, ILFs, mucosal immunoglobulin (Ig)A production, oral tolerance, and pathology in rodent gastritis and colitis models. ${ }^{5,6}$

The current story warmed up in 2010 when a team led by Alexei Tumanov and Yang-Xin Fu followed up on an observation made by Thomas Spahn and colleagues in 2004 that mice lacking the surface LT (the LT $\alpha / \beta$ trimeric ligand) or its receptor, LT $\beta$ R, readily succumbed to a Citrobacter rodentium infection. ${ }^{7,8}$ C. rodentium infection is often used to explore the inflammatory effects of an enteric pathogen and the host's immune response. They found that LT expressed by ROR $\gamma t+$ lymphocytes, i.e., ILC, was critical for lymphocyte communication to the epithelium and the recruitment of neutrophils to the damaged barrier. Direct signaling of LT ligand on lymphocytes to receptor-positive epithelial cells is certainly plausible based on the studies of lymphotoxin- $\beta$ receptor (LT $\beta R$ ) agonism in colorectal carcinoma cells or hepatocytes in hepatitis. ${ }^{9,10}$ An important prequel to this saga was written in 2008 by Wenjun Ouyang's group at Genentech

${ }^{1}$ Department of Immunobiology, Biogen Idec, Cambridge, Massachusetts, USA. Correspondence: JL Browning (jeff.browning@biogenidec.com) 


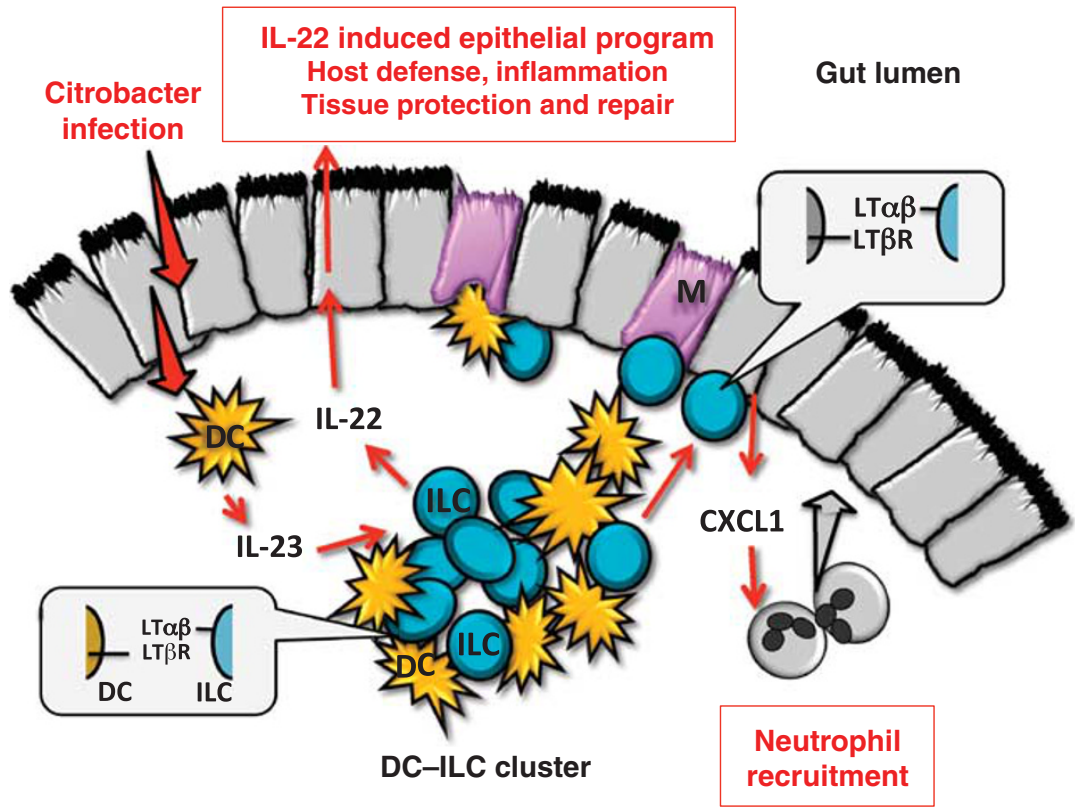

Lamina propria

Figure 1 Schematic illustration of the proposed events following C. rodentium infection. Infection is sensed by dendritic cells (DCs) causing them to secrete interleukin (IL)-23. IL-23 triggers the innate lymphoid cells (ILCs) to make the IL-22 that can directly engage receptors on epithelial cells and initiate an epithelial response program. This program includes host defense mechanisms such as anti-microbial protein release, e.g., REG III $\beta$ and $\gamma$, secretion of inflammatory cytokines and chemokines as well as various tissue protection and repair elements. Host defense to this pathogen absolutely relies on the induction of this IL-22-initiated epithelial response. Selective deletion of lymphotoxin- $\beta$ receptor expression (LT $\beta R$ ) in DC or in epithelial cells revealed roles for the receptor in both cell types. Therefore, direct ILC-DC and ILC-epithelial dialogs have been postulated. ILCs exist as clusters with DC in the villi in organized structures such as isolated lymphoid follicles and colonic follicles with M-cell links to the lumen.

in San Francisco, where they showed that early secretion of IL-22 was critical for survival following C. rodentium infection, and, moreover, IL-23 drove the expression of IL-22. ${ }^{11}$ IL-22 triggers a complex gene expression program in epithelial cells with proinflammatory, chemokine secretion, production of anti-microbial proteins, and tissue repair components. Release of anti-microbial proteins such as RegIII $\gamma$ helps to control the initial bacterial invasion. It was a quick leap in subsequent papers by both groups to show that IL-22 production was impaired in mice deficient in the LT pathway. ${ }^{12-14}$

In both studies, the LT $\alpha / \beta$ ligand and not the alternative LT $\beta R$ ligand, LIGHT, was found to be responsible for the IL23/IL-22 effects and, moreover, LT ligand expression by ROR $\gamma \mathrm{t}+$ ILC was essential. While these rodent events were clearly LIGHT-independent, LIGHT has considerable history in the gut and its involvement in other mucosal settings may be more dramatic such as seen in the recent observations in the lung. ${ }^{15}$ IL-22 is made by multiple cell types; however, the LTi is the dominant source in this setting. 2,16 LT $\beta R$ is typically not present on lymphocytes, indicating indirect regulation of IL-22 production from LTi. Upon dissection, LT $\beta$ R signaling was required to induce the IL-23 that triggers the IL-22 response. ${ }^{12}$ As dendritic cells are dominant producers of IL-23, an LT-dependent dialog between dendritic cells (DC) and ILC was postulated. Whether the role of LT $\beta$ R signaling is strictly limited to modulating DC production of IL-23 is less certain. A DC selective deletion of LT $\beta$ R resulted only in a partial exacerbation of $C$. rodentium infection and a modest twofold decrease in IL-23 production. ${ }^{12}$ Moreover, the earlier work showed that LT $\beta R$ on both epithelium and hematopoietic cells was critical. ${ }^{8}$ The work by Ota et al. ${ }^{13}$ focused on the organized lymphoid architecture in the colon, i.e., CLP and ILF and the hypertrophy of these tissues that occurs following $C$. rodentium infection. Blockade of LT $\beta$ R signaling in mice infected with C. rodentium reduced the size and organization of these structures, an observation also made previously in another colitis setting. ${ }^{17}$ Therefore, the role of LT $\beta R$ is likely a combination of maintaining TLT organization along with both ILC-DC and ILCepithelial communication. These events could be intertwined with the control of chemokine-induced positioning, perhaps involving CCL20 production.

The ability of LT $\beta R$ signaling to orchestrate cell trafficking and positioning is well defined in both secondary and TLTs in normal and reactive states. It appears that there are several routes to TLT formation and organization including ROR $\gamma \mathrm{t}+$ ILC dependent and independent as well as LT dependent and independent mechanisms. In general, the formation of large TLT is LT-dependent, but recently, and of relevance to the gut, Th17 cells were shown to trigger TLT formation in the lung. ${ }^{18}$ Somewhat surprisingly, in the Ouyang study, IL-22 administration was able to restore robust TLT in the colon even in the presence of LT $\beta$ R blockade, suggesting that IL-22 can directly impact organization. It will be interesting to see how these IL-22-mediated events translate in colitis settings as infection models have considerable additional complexity. For example, the net impact of $C$. rodentium on host survival is a gemisch of colitis, effective host immune response, systemic spread of the infection and the kinetics of bacterial clearance. Pathogen clearance in LT-deficient mice is impaired in many studies, presumably reflecting alterations in basic adaptive immunology or efficient antigen sensing. In general, the questions of whether the lack of LT $\beta R$ signaling led to an inefficient or slowed immune response and/or an unchecked inflammatory response remain poorly explored. Second, LT $\beta R$ signaling is central to the differentiation of high endothelial venules and display of addressins in both secondary and tertiary settings. Therefore, the trafficking of various cells into the reactive sites should be altered in the absence of LT $\beta$ R signaling. Reduced trafficking could be anti-inflammatory, 
yet also impair the host response to the pathogen. Finally, in several lymphoid tissues, the fibroblastoid reticular networks are affected by LT $\beta R$ signaling, and this element could be an important factor in the host response to $C$. rodentium. Indeed, LT $\beta R$ expression on mucosal nonhematopoietic cells is required for the development of gut IgA production. ${ }^{19}$

These data point toward a more intimate relationship between the ILC and DC in the mucosal niches, and there were some clues from prior studies. In what was a rather perplexing body of work; DC homeostasis or the maintenance of normal numbers of DCs in some secondary lymphoid organs was at least partially LT-dependent. ${ }^{20}$ Recently, the differentiation of a DC subset in the spleen and gut was found to require Notch2 receptor signaling in an LT $\beta R$ dependent manner. ${ }^{21}$ Intriguingly, the differentiation of ROR $\gamma t+$ ILC in the periphery is also Notch2-dependent and it will be interesting to learn if these two events are related. ${ }^{22,23}$ More poignantly, the idea of a DC-ILC dialog resonates well with a dissection of the roles of LT $\beta R$ and CD40 signaling in DC during adaptive T-cell responses, as elucidated in the work of Jen Gommerman and colleagues ${ }^{24}$ in Toronto. In fact, their work started with the observation that DC-T cell clusters were disrupted following pharmacological blockade of LT $\beta R$ signaling. DC licensing for an adaptive T-cell response was found to require LT $\beta \mathrm{R}$ signaling, and this function was non-redundant with CD40 signaling even though CD40 signaling is classically associated with DC licensing/maturation. Does the gut simply represent a similar dialog between more constitutively LT-positive ILC and $D C$ ? Non-canonical nuclear factor- $\kappa \mathrm{B}$ signaling is crucial for DC maturation/ licensing and it is reasonable that LT $\beta$ R signaling could provide this element. ${ }^{25}$

Human inflammatory bowel disease (IBD) is viewed as a deviation from the normal balance between the microbiome and the mucosal immune system with a continuing cycle of inflammation-triggered epithelial damage and excessive immune activation. How would an LT $\beta R$ inhibitory agent fare in this setting? It has been posited that many experimental therapies for IBD have been limited in efficacy because a larger swath of the immunology needs to be brought under control. ${ }^{26}$ The exaggerated dextran sulfate sodium colitis in the genetic absence of ROR $\gamma t$ and hence lack of ILC certainly highlights the many roads to TLT formation and inflammation. LT $\beta$ R inhibition attenuates disease in a wide range of mouse colitis models including an ROR $\gamma$ t-independent setting. ${ }^{4,27}$ It can affect a broad range of immunological events, including the production of certain homeostatic chemokines, neutrophil-attracting chemokines released by epithelial cells, vascular addressin expression, reticular network specialization, the maintenance of large TLT, and now modulation of IL-22-triggered epithelial defense/repair programs. The consequences of IL-22 inhibition are difficult to forecast as IL-22 has both proinflammatory and repair roles depending on the organ and even differs between different IBD models. ${ }^{2}$ Thus, there appears to be some pieces missing in this puzzle, such as a temporal component to the expression of IL-17/IL-22 or perhaps the expression of the soluble IL-22 inhibitory receptor, Il22ra2, can resolve this conundrum. Whatever the exact details surrounding IL-22's protective effects, LT $\beta R$ signaling appears to have a fundamental organizing role in the center ring of this circus. It will be exciting to see whether this LT $\beta R$ core function reflects control of the spatial organization of the DC-ILC clusters or more direct ILC-DC or ILCepithelial signaling events. It is possible in IBD that LT $\beta R$ inhibition could reduce the amount of gut immunological activity at multiple levels, break the pathological cycle of commensal imbalance and barrier breach, and allow a "time-out" for the immune system to reset.

\section{DISCLOSURE}

JLB is employed by Biogen Idec, which has had an interest in the LT $\beta R$ pathway.

C 2012 Society for Mucosal Immunology

\section{REFERENCES}

1. Aparicio-Domingo, P. \& Cupedo, T. Rorgammat+ innate lymphoid cells in intestinal homeostasis and immunity. J. Innate Immun. 3, 577-584 (2011).
2. Sonnenberg, G.F., Fouser, L.A. \& Artis, D. Border patrol: regulation of immunity, inflammation and tissue homeostasis at barrier surfaces by IL-22. Nat. Immunol. 12, 383-390 (2011).

3. van de Pavert, S.A. \& Mebius, R.E. New insights into the development of lymphoid tissues. Nat. Rev. Immunol. 10, 664-674 (2010).

4. Browning, J.L. Inhibition of the lymphotoxin pathway as a therapy for autoimmune disease. Immunol. Rev. 223, 202-220 (2008).

5. Wang, J. \& Fu, Y.X. Tumor necrosis factor family members and inflammatory bowel disease. Immunol. Rev. 204, 144-155 (2005).

6. Spahn, T.W., Muller, M.K., Domschke, W. \& Kucharzik, T. Role of lymphotoxins in the development of Peyer's patches and mesenteric lymph nodes: relevance to intestinal inflammation and treatment. Ann. NY Acad. Sci. 1072, 187-193 (2006).

7. Spahn, T.W. et al. The lymphotoxin-beta receptor is critical for control of murine Citrobacter rodentium-induced colitis. Gastroenterology 127, 1463-1473 (2004).

8. Wang, Y. et al. Lymphotoxin beta receptor signaling in intestinal epithelial cells orchestrates innate immune responses against mucosal bacterial infection. Immunity $\mathbf{3 2}$, 403-413 (2010).

9. Lukashev, M. et al. Targeting the lymphotoxinbeta receptor with agonist antibodies as a potential cancer therapy. Cancer Res. 66, 9617-9624 (2006).

10. Haybaeck, J. et al. A lymphotoxin-driven pathway to hepatocellular carcinoma. Cancer Cell 16, 295-308 (2009).

11. Zheng, Y. et al. Interleukin-22 mediates early host defense against attaching and effacing bacterial pathogens. Nat. Med. 14, 282-289 (2008).

12. Tumanov, A.V. et al. Lymphotoxin controls the IL-22 protection pathway in gut innate lymphoid cells during mucosal pathogen challenge. Cell Host Microbe 10, 44-53 (2011).

13. Ota, N. et al. IL-22 bridges the lymphotoxin pathway with the maintenance of colonic lymphoid structures during infection with Citrobacter rodentium. Nat. Immunol. 12, 941-948 (2011).

14. Spits, $H$. Another armament in gut immunity: lymphotoxin-mediated crosstalk between innate lymphoid and dendritic cells. Cell Host Microbe 10, 3-4 (2011).

15. Doherty, T.A. et al. The tumor necrosis factor family member LIGHT is a target for asthmatic airway remodeling. Nat. Med. 17, 596-603 (2011).

16. Sawa, S. et al. RORgammat+ innate lymphoid cells regulate intestinal homeostasis by integrating negative signals from the symbiotic microbiota. Nat. Immunol. 12, 320-326 (2011).

17. Dohi, T. et al. Elimination of colonic patches with lymphotoxin beta receptor-lg prevents Th2 cell-type colitis. J. Immunol. 167, 2781-2790 (2001).

18. Rangel-Moreno, J. et al. The development of inducible bronchus-associated lymphoid tissue depends on IL-17. Nat. Immunol. 12, 639-646 (2011).

19. Kang, H.S. et al. Signaling via LTbetaR on the lamina propria stromal cells of the gut is required for IgA production. Nat. Immunol. 3 , 576-582 (2002).

20. De Trez, C. \& Ware, C.F. The TNF receptor and Ig superfamily members form an integrated 
signaling circuit controlling dendritic cell homeostasis. Cytokine Growth Factor Rev. 19, 277-284 (2008).

21. Lewis, K.L. et al. Notch2 receptor signaling controls functional differentiation of dendritic cells in the spleen and intestine. Immunity 35 , 780-791 (2011).

22. Possot, C. et al. Notch signaling is necessary for adult, but not fetal, development of RORgammat(+) innate lymphoid cells. Nat. Immunol. 12, 949-958 (2011).
23. Lee, J.S. et al. AHR drives the development of gut ILC22 cells and postnatal lymphoid tissues via pathways dependent on and independent of Notch. Nat. Immunol. 13, 144-151 (2012).

24. Gommerman, J.L. \& Summers Deluca, L. LTbetaR and CD40: working together in dendritic cells to optimize immune responses. Immunol. Rev. 244, 85-98 (2011).

25. Hofmann, J., Mair, F., Greter, M., SchmidtSupprian, M. \& Becher, B. NIK signaling in dendritic cells but not in $\mathrm{T}$ cells is required for the development of effector T cells and cellmediated immune responses. J. Exp. Med. 208, 1917-1929 (2011).

26. Macdonald, T.T. Inside the microbial and immune labyrinth: totally gutted. Nat. Med. 16, 1194-1195 (2010).

27. Lochner, M. et al. Microbiota-induced tertiary lymphoid tissues aggravate inflammatory disease in the absence of RORgammat and LTi cells. J. Exp. Med. 208, 125-134 (2011). 